\title{
Laparoscopic Strassman's metroplasty for bicornuate uterus
}

\author{
Sanket Pisat • Benedikt Tas $\cdot$ Bruno van Herendael
}

Received: 3 December 2008 / Accepted: 29 December 2008 / Published online: 30 January 2009

(C) Springer-Verlag 2009

\begin{abstract}
The authors report the case of a 28-year-old nulliparous woman with a bicornuate uterus and one previous second trimester pregnancy loss, in whom investigations for other probable causes of abortion like genetic, infective, hormonal, and immunological were negative. A laparoscopic metroplasty was performed by Strassman's method. Second-look hysteroscopy and laparoscopy, which was performed 7 months later, revealed a single uniform cavity with a median muscular ridge, which resembled an arcuate uterus. Pelvic adhesions were noted between the small bowel, omentum, and posterior wall of the uterus, along with pelvic endometriosis and a chocolate cyst of the left ovary. Adhesiolysis, cyst excision, and fulgration of endometriotic deposits were carried out, which were followed by the application of an adhesion barrier.
\end{abstract}

Keywords Laparoscopic metroplasty · Bicornuate uterus . Strassman's metroplasty $\cdot$ Mullerian abnormalities

S. Pisat $\cdot$ B. Tas $\cdot$ B. van Herendael $(\square)$

Endoscopic Training Centre Antwerp (ETCA),

Ziekenhuis Netwerk Antwerpen [ZNA] Stuivenberg,

Lange Beeldekensstraat 267,

2060 Antwerp, Belgium

e-mail: bruno.vanherendael@beline.be

URL: www.etca.be

S. Pisat

e-mail: sanket.pisat@gmail.com

URL: www.etca.be

B. Tas

e-mail: benedictus.tas@zna.be

URL: www.etca.be

B. van Herendael

Obstetrics and Gynecology Department, Università dell'Insurbia,

Varese, Italy

\section{Introduction}

The incidence of uterine malformations in the general population is about $4.3 \%$, and in patients with recurrent pregnancy loss, it is about $13 \%$ [1]. Bicornuate uteri constitute around $25 \%$ of Mullerian anomalies [2]. The pregnancy outcome of patients with untreated bicornuate uterus is poor, with reported term delivery rates of up to $62.5 \%$, with early miscarriages and preterm deliveries being quite common [3]. Conventional transabdominal metroplasty has been shown to significantly improve the pregnancy outcome in patients with bicornuate uterus [4]. Laparoscopic metroplasty, though technically difficult, offers the distinct advantages of the laparoscopic approach over laparotomy. There are, till date, very few published reports of laparoscopic metroplasties, the first one being reported in January 2006 [5]. In this case report, the authors describe a laparoscopic metroplasty for a bicornuate uterus, with good postoperative results confirmed by second-look hysterolaparoscopy.

\section{Case report}

A 28-year-old woman with a history of one previous second trimester pregnancy loss at 17 weeks of gestation was referred for management. On physical examination, she had normal physical and secondary sexual characteristics. External genital organs were normal. An ultrasound examination revealed a bicornuate uterus with two symmetrical uterine horns and a single cervix. Both ovaries were normal. On inquiry, the patient gave a history of similar malformations in other members of her family. A renal ultrasound was done to rule out associated anomalies of the urinary system. Investigations for other probable 
causes of abortion like genetic, infective, hormonal, and immunological were negative.

\section{Materials and methods}

Operative procedure

Diagnostic hysterolaparoscopy with laparoscopic metroplasty was performed in the early proliferative phase. A single dose of antibiotic (third generation cephalosporins) was given intravenously at the start of the surgery. Antiembolic stockings were used for thromboembolism prophylaxis. A written informed consent of the patient and her husband was taken after explaining the procedure, its possible complications, and impact on her reproductive outcome.

After placing the patient in lithotomy position, diagnostic hysteroscopy and laparoscopy was done to confirm the diagnosis of bicornuate uterus (Fig. 1). Both horns of the uterus were found to be of approximately the same size and shape. Both horns were functional and communicating at the isthmus. Both tubal ostia, one in each horn, appeared normal. Bilateral tubal patency was confirmed by methylene blue test.

The surgical procedure of laparoscopic Strassman's metroplasty

A 2.5-cm-long intraumbilical incision was taken, and a $20 \mathrm{~cm}$ Veress needle was inserted through it. Pneumoperitoneum was created using prewarmed and humidified $\mathrm{CO}_{2}$ at a rate of $4.5 \mathrm{~L} / \mathrm{min}$. The temperature of the $\mathrm{CO}_{2}$ gas at abdominal entry was $35^{\circ} \mathrm{C}$ at a preset pressure of $20 \mathrm{~mm}$ $\mathrm{Hg}$ using a special $\mathrm{CO}_{2}$ insufflator (Thermoflator, Karl

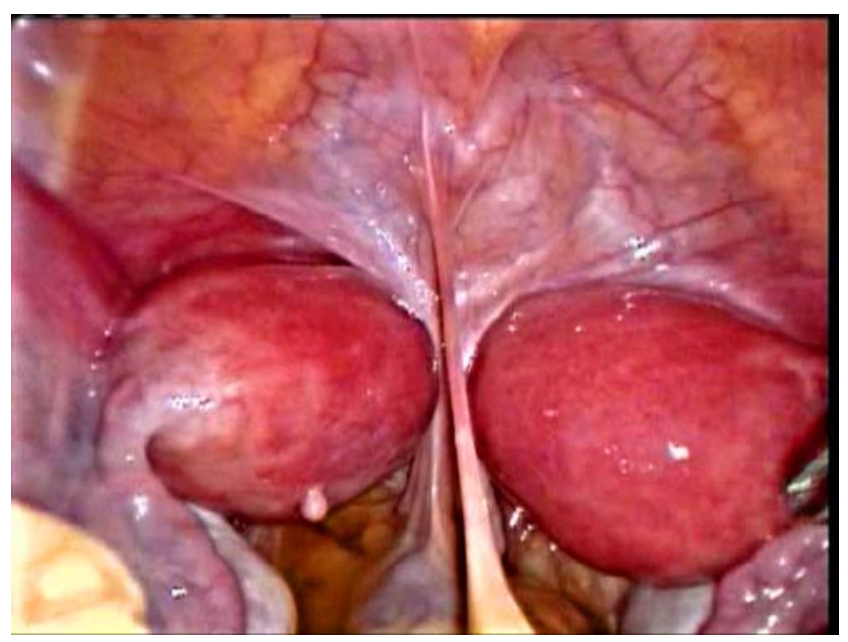

Fig. 1 Bicornuate uterus with adhesion band between bladder and rectum
Storz Gmbh \& Co. KG, Tuttlingen, Germany). An $11 \mathrm{~mm}$ trocar with reduction to 10 HiCap (Karl Storz Gmbh \& Co. $\mathrm{KG})$ was inserted through this incision, and the abdominal cavity was inspected using a zero degree telescope. Two $5 \mathrm{~mm}$ trocars were placed in the right and left lower quadrants of the abdomen, medial to the inferior epigastric vessels, and a third $10 \mathrm{~mm}$ trocar was placed in the midline $15 \mathrm{~cm}$ above the pubic symphysis. An adhesion band was seen extending from the bladder to the rectum, between the two uterine horns. The fallopian tubes and ovaries were normal. There were no adhesions in the ovarian fossa. Superficial endometriotic deposits were seen in the pouch of Douglas, which were fulgrated with bipolar cautery. Using bipolar current, the adhesion band was cauterized and then cut with scissors.

Epinephrine solution $(30 \mathrm{~mL})$ in a dilution of $1 \mathrm{mg}$ epinephrine in $100 \mathrm{~mL}$ saline was infiltrated subserosally along the medial aspect of both uterine horns. Using a monopolar cautery at $120 \mathrm{~W}$ pure cutting current, an incision deep enough to cut the myometrium was taken. This incision started from the supero-medial aspect of each uterine horn, about $2 \mathrm{~cm}$ medial and caudal to the origin of the fallopian tube and extended along the medial aspect of the horn up to its base. For controlling myometrial bleeding, bipolar cautery at $80 \mathrm{~W}$ coagulating current was used. The endometrial cavity was then opened along the same line using scissors (Fig. 2). The uterus was manipulated using a blunt-tipped metallic uterine sound. Initially, the sound was inserted into the cervix up to the base of the uterine horns to push up the base while taking myometrial incision with monopolar cautery. Then, the sound was inserted into one uterine horn at a time to stabilize it before cutting the endometrium with scissors over the instrument. $\mathrm{CO}_{2}$ inflow was increased to $20 \mathrm{~L} / \mathrm{min}$ to prevent loss of pneumoperitoneum. Although there was a loss of pneumo-

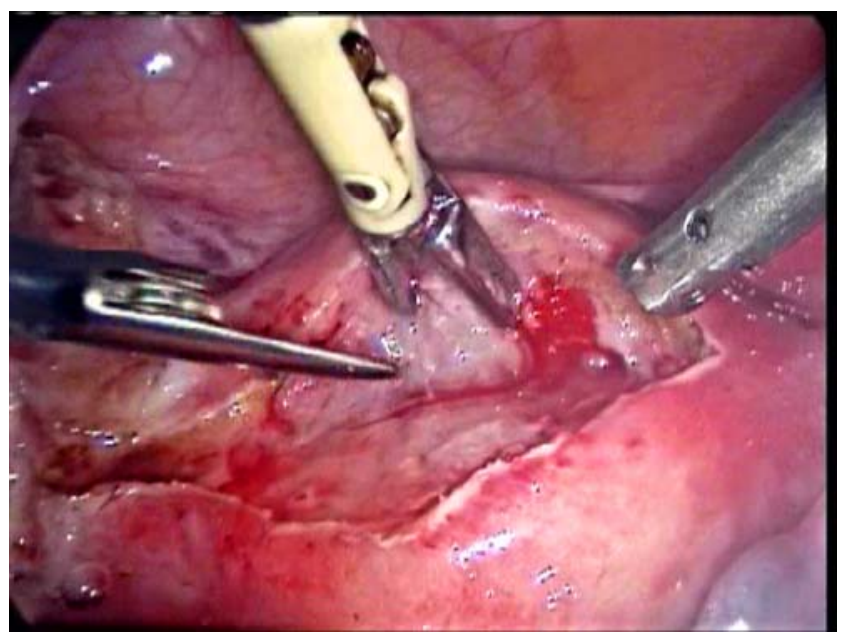

Fig. 2 Opening endometrial cavity with scissors after myometrial incision with unipolar cautery 
peritoneum after opening the endometrium, the loss was slow and gradual due to presence of the cervix. Increasing $\mathrm{CO}_{2}$ inflow was enough to nullify this gradual loss and maintain good abdominal distension for surgery. Hence, vaginal packing was not necessary.

The opposing myometrial edges were sutured using interrupted sutures of 0 polyglactin 910 (Vicryl; Ethicon, Somerville, NJ, USA), taking successive sutures on the anterior and posterior walls. Vicryl no. $0,90 \mathrm{~cm}$ in length, on a $26 \mathrm{~mm}$ half circle round body needle was used. Care was taken to exclude the endometrium. One layer of inverting sutures was used, thereby burying the knots in the myometrium to minimize postoperative adhesion formation. After placing two sutures each on the anterior and posterior wall at the base, two square sutures of Vicryl no. 1 (second layer) were placed at the fundus, anteriorly and posteriorly. The purpose of these sutures was to achieve good approximation of the opposing walls, achieve hemostasis, and to enable manipulation of the uterus, thereby making it easier to take the rest of the sutures. The peritoneal cavity was irrigated with saline solution and hemostasis was confirmed (Fig. 3). The uterus and peritoneal cavity were then irrigated with $1 \mathrm{~L}$ of Icodextrin 4\% solution (Adept; Baxter Healthcare S.A., Belgium), which was left inside the peritoneal cavity to minimize postoperative adhesion formation. The infraumbilical port was closed with a delayed absorbable monofilament suture in two layers. Skin closure of all ports was done using cyanoacrylate skin adhesive (Dermabond; Ethicon). The intraoperative blood loss was $100 \mathrm{~cm}^{3}$, and the duration of surgery was $3 \mathrm{~h}$.

Postoperative course and second-look hysterolaparoscopy

The patient had an uneventful recovery and was discharged on the third postoperative day. Antibiotics were continued

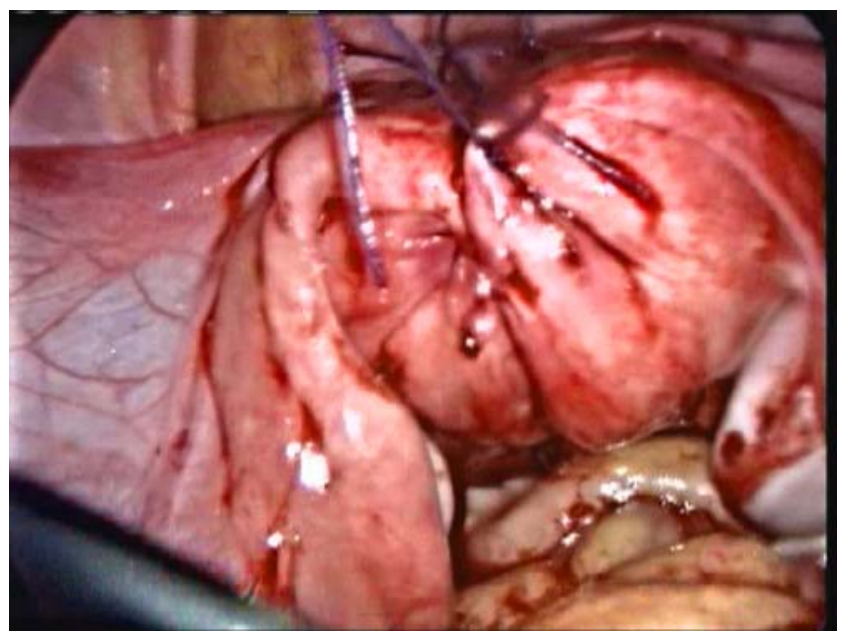

Fig. 3 Reconstructed uterus after completion of suturing for 5 days. Barrier contraceptives were prescribed. She was advised to follow up after 4 months for repeat hysterolaparoscopy, but it was postponed by the patient for family causes.

A repeat hysteroscopic and laparoscopic examination was performed 7 months later. Hysteroscopy using the Bettochi vaginoscopy technique and a $3.5 \mathrm{~mm}$ rigid through flow hysteroscope (Karl Storz Gmbh \& Co. KG) showed a uniform, spacious uterine cavity with a median muscular ridge less than $1 \mathrm{~cm}$ in size, resembling an arcuate uterus, for which no treatment was felt necessary (Fig. 4). Endometrium and tubal ostia were normal. No synechiae were seen. On laparoscopy, a normal uterus with a uniform external contour and perfect bilateral symmetry was seen. There were filmy adhesions between both the omentum, small bowel, and the posterior uterine wall (Fig. 5). A chocolate cyst was seen in the left ovary. The adhesions were coagulated and cut, and the chocolate cyst was drained and then excised. The raw areas were covered with an adhesion barrier substance (SprayShield; Covidien, Mansfield, MA, USA) to prevent recurrence of adhesions (Fig. 6).

\section{Discussion}

Transabdominal metroplasty by laparotomy and its impact on reproductive capability have been extensively studied by various authors $[4,6]$. This procedure has been shown to significantly improve the obstetric outcome in women with recurrent abortions or premature delivery before surgery [4]. The term pregnancy rates after unification procedure have approached $80 \%$ to $85 \%$ [6]. Although there are as yet

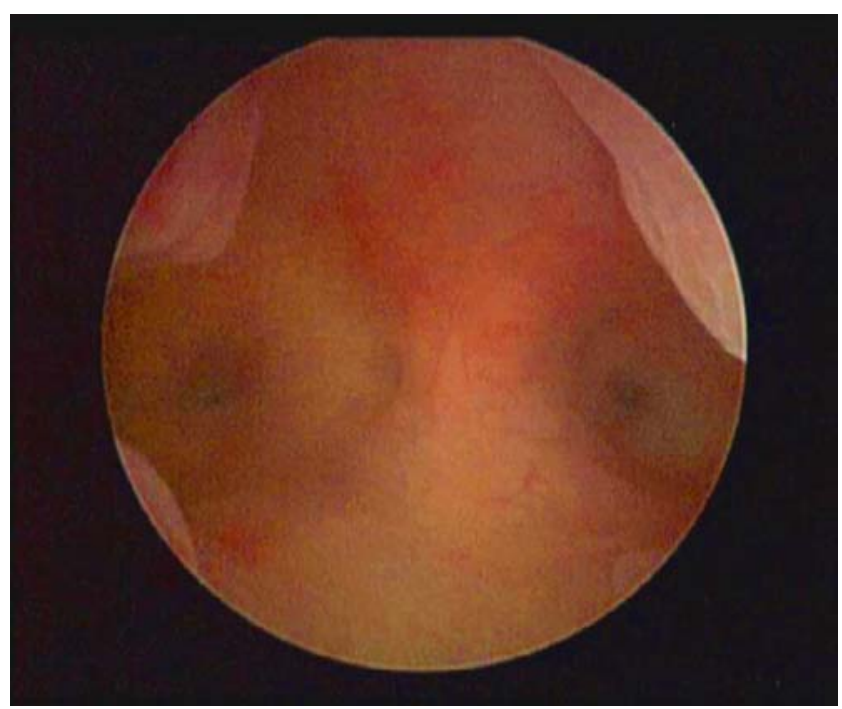

Fig. 4 Second-look hysteroscopy showing spacious cavity with median muscular ridge 


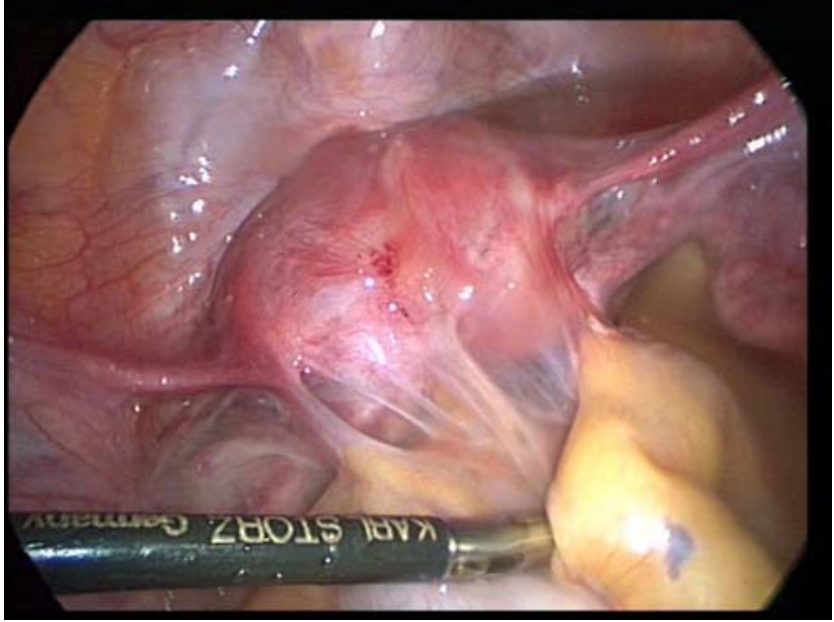

Fig. 5 Second-look laparoscopy showing filmy adhesions between omentum, small bowel, and uterus

few reports of laparoscopic metroplasty, initial results are encouraging. Authors have reported minimal peritoneal adhesions, good restoration of uterine anatomy, and a spacious, uniform cavity $[5,7]$.

Although there is limited experience with the laparoscopic correction of Mullerian anomalies, the laparoscopic approach could be a viable alternative to abdominal Strassman's metroplasty. Reduced tissue handling and drying, which are inherent in laparoscopy, may contribute to reduced adhesion formation after laparoscopic surgery [8]. Also, early ambulation, reduced hospital stay, quicker wound healing, and a more cosmetic scar are some of the obvious advantages of the laparoscopic approach over laparotomy.

However, some issues are of concern. Strassman's metroplasty is technically quite challenging when performed through the laparoscopic route [5]. Precise suturing to achieve good approximation of the anterior and posterior

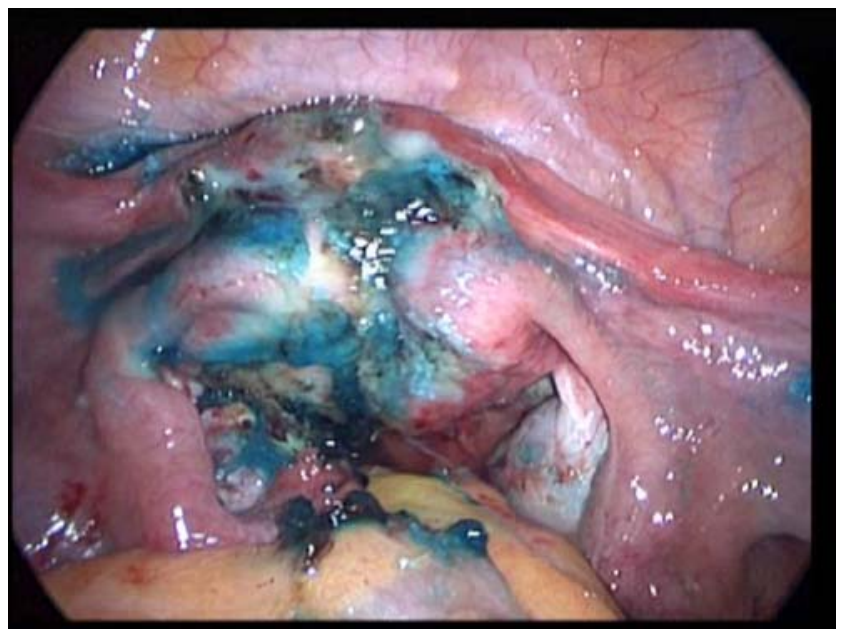

Fig. 6 After adhesiolysis and spraying of adhesion barrier walls is difficult and tiresome. Manipulation of the needle holders while suturing the base of the posterior uterine wall can be extremely difficult [5]. Bleeding through the cut edges of the uterine wall can interfere with the suturing process. The laparoscopic metroplasty procedure differs somewhat from conventional abdominal metroplasty. Fewer sutures were used to approximate the uterine walls than are needed in conventional metroplasty. The authors used no. 0 Vicryl, which is thicker and stronger than finer sutures that are otherwise used, as these sutures tend to break easily during intracorporeal knot tying. While suturing the uterine walls, interrupted inverting sutures were used. The needle was first passed into the myometrium adjacent to the endometrial lining, to exit just below the serosa, on the left horn. The needle then passed through the myometrium of the right horn, first subserosally and exited adjacent to endometrium. On tying this suture intracorporeally, the endometrial edges were apposed perfectly, and the knot was buried in the myometrium. Inverting the myometrium in this way prevents growth of endometrial glands in the uterine scar [5,9]. Placing all knots within the cavity or on the serosa of the uterus would aggravate formation of intrauterine synechiae and external adhesions, respectively.

The second issue is that of achieving meticulous hemostasis, vis-à-vis with the excessive use of current, which may cause weakening of the scar. Most authors have described opening the myometrium using a monopolar needle. The myometrium is damaged to a certain extent using this technique, which causes weakening of the scar, but it gives good hemostasis [5, 7]. The myometrial edges must not be sutured under tension, and hematoma formation in the wall should be avoided. Suturing myometrial edges under tension increases the chances of necrosis of the uterine musculature. Good approximation without tension will prevent hematoma formation in the uterine musculature. It will also reduce the likelihood of healing by secondary intent, which could make the uterine wall fragile during pregnancy $[5,7,9]$.

The third issue is the integrity of the scar and its performance in labor. Reports show a good scar integrity in myomectomy cases wherein the capsule of the fibroid is opened with a monopolar needle [9]. During the second stage of labor, uterine contractions reach $80-100 \mathrm{mmHg}$. To evaluate uterine compliance in labor, authors have elevated intrauterine pressure up to $150 \mathrm{~mm} \mathrm{Hg}$ with a continuous positive pressure flow of $5 \%$ dextrose solution and confirmed that the uterine wall tolerated it [7]. Recent studies have shown that uterine rupture during pregnancy after laparoscopic myomectomy is rare, and vaginal birth after laparoscopic myomectomy appears to be safe in selected patients who meet certain criteria [10]. However, it must be remembered that in Strassman's metroplasty, the uterine cavity is opened over its entire length and the 
myometrium is incised longitudinally. These patients will definitely have an increased risk of scar rupture in late pregnancy, as compared to myomectomy, where only a partial incision of the myometrium overlying the myoma is taken, without opening the cavity in most cases. It is worthwhile to note that studies have shown term delivery rates in bicornuate uteri to be up to $62.5 \%$ [3]. Because a laparoscopic metroplasty is an invasive procedure and poses an increased obstetric risk in late pregnancy, it is only indicated in case of proven poor obstetric outcome and in absence of other reasons for recurrent miscarriage.

The fourth issue is that of postoperative adhesion formation. Although the minimally invasive endoscopic approach has been shown to be less adhesiogenic than traditional surgery, at least with regard to selected procedures, it does not totally eliminate the problem [11]. Minimal adhesion formation after laparoscopic metroplasty for bicornuate and didelphic uteri has been reported in previous studies [5, 7]. In our case, the co-existent endometriosis could have been an important factor in the development of postoperative adhesions. A higher incidence of endometriosis has been reported in patients with a septate uterus [12], and this may also be true for other Mullerian anomalies.

The temperature and dryness of the gas used during laparoscopy cause physical and chemical alterations in the peritoneum, leading to postoperative adhesion formation. Damage to the peritoneal surfaces during laparoscopy can be minimized when the gas stream is kept warm and moist [13]. Liquid agents, which can be instilled in the abdominal cavity, prevent adhesions by a combination of hydroflotation and barrier principles [14]. A study using Icodextrin 4\% solution conducted in 150 centers across six European countries concluded that the solution was well tolerated and easy to use for the reduction of adhesion formation following gynecological surgery [15].

The use of certain sprayable hydrogel adhesion barriers has also been shown to be effective in reducing postoperative adhesion severity [16]. The authors are of the opinion that in cases where sutures have been taken on the surface of the uterus, using a sprayable hydrogel adhesion barrier results in lesser postoperative adhesions between the uterus and other organs, like the bowel and omentum. This is because the gel solidifies after spraying, has excellent tissue adherence, remains strongly adherent to the site of application for 5 to 7 days, and hence is ideal for the prevention of site-specific adhesions [16]. Hence, the effect of the cut ends of sutures, which would otherwise stimulate adhesion formation due to a foreign body reaction, is minimized. The hope is that an improved formula, which is highly effective, will be developed soon. At their institute, the authors now have a routine policy of using prewarmed, humidified $\mathrm{CO}_{2}$ for every laparoscopic procedure. Adhe- sion barrier spray (SprayShield; Covidien) is routinely used after every myomectomy and adhesiolysis.

It is worthwhile to note that the new uterine cavity after a conventional transabdominal metroplasty appears smaller than that of a normal uterus [6]. However, this does not seem to be applicable for the present case. The final size of the uterine cavity seems to be relatively unimportant to reproductive capability; uterine symmetry appears to be a more important factor [6].

\section{Conclusion}

In conclusion, laparoscopic Strassman's metroplasty seems to be a viable alternative to the traditional transabdominal approach by laparotomy. This procedure can be kept as a surgical option in symptomatic cases that require uterine unification for bicornuate uteri. More long-term studies, as regards scar integrity and reproductive outcome, are needed to establish a comparison over the laparotomy approach.

Declaration The authors Dr Sanket Pisat, Dr Benedikt Tas, and Prof Dr Bruno van Herendael hereby declare that the surgery described in this manuscript complies with the current laws of Belgium.

Conflict of interest The corresponding author certifies that there is no actual or potential conflict of interest in relation to this article.

\section{References}

1. Grimbizis GF, Camus M, Tarlatzis BC, Bontis JN, Devroey P (2001) Clinical implications of uterine malformations and hysteroscopic treatment results. Hum Reprod Update 7:161-174

2. Porcu G, Heckenroth H (2005) Uterine abnormalities and infertility. EMC Gynecol Obstet 2:185-197

3. Raga F, Bauset C, Remohi J, Bonilla-Musoles F, Simon C, Pellicer A (1997) Reproductive impact of congenital Mullerian anomalies. Hum Reprod 12(10):2277-2281

4. Papp Z, Mezei G, Gávai M, Hupuczi P, Urbancsek J (2006) Reproductive performance after transabdominal metroplasty: a review of 157 consecutive cases. J Reprod Med 51(7):544-552

5. Sinha R, Mahajan C, Hegde A, Shukla A (2006) Laparoscopic metroplasty for bicornuate uterus. J Minim Invasive Gynecol 13:70-73 doi:10.1016/j.jmig.2005.11.004

6. Rock JA (1997) Surgeries for anomalies of the mullerian ducts. In: Rock JA, Thompson JD (eds) The Linde's operative gynaecology. 8th edn. Lippincott-Raven, Philadelphia, pp 687729

7. Alborzi S, Asadi N, Zolghadri J, Alborzi S, Alborzi M (2008) Laparoscopic metroplasty in bicornuate and didelphic uteri. Fertil Steril. doi:10.1016/j.fertnstert.2008.04.025

8. Stringer NH, Walker JC, Meyer PM (1997) Comparison of 49 laparoscopic myomectomies with 49 open myomectomies. J Am Assoc Gynecol Laparosc 4:457-464 
9. Sinha RY, Hegde A, Warty N et al (2003) Laparoscopic excision of very large myomas. J Am Assoc Gynecol Laparosc 10:461468

10. Kumakiri J, Takeuchi H, Itoh S, Kitade M, Kikuchi I, Shimanuki $\mathrm{H}$ et al (2008) Prospective evaluation for the feasibility and safety of vaginal birth after laparoscopic myomectomy. J Minim Invasive Gynecol 15:420-424 doi:10.1016/j-jmig.2008.04.008

11. Nappi C, Sardo AD, Greco E, Guida M, Bettocchi S, Bifulco G (2007) Prevention of adhesions in gynaecological endoscopy. Hum Reprod Update 13(4):379-394 doi:10.1093/humupd/ dm1061

12. Nawroth F, Rahimi G, Nawroth C, Foth D, Ludwig M, Schmidt T (2006) Is there an association between septate uterus and endometriosis? Hum Reprod 21(2):542-544 doi:10.1093/humrep/ dei344
13. Ott DE (2004) The peritoneum and the pneumoperitoneum: a review to improve clinical outcome. Gynecol Surg 1(2):101-106

14. van Herendael B (2004) Adhesions and their importance in gynecological surgery. The importance and the timing of secondlook laparoscopy. In: Marana R, Busacca M, Zupi E (eds) World meeting on mininally invasive surgery in gynecology. Medimond S.r.1.-Monduzzi Editore International Proceedings Division, Bologna, pp 1-8

15. Sutton C, Minelli L, Garcia E, Korell M, Pouly JL, Pados G et al (2005) Use of Icodextrin 4\% solution in the reduction of adhesion formation after gynaecological surgery. Gynecol Surg 2:287-296

16. Mettler L, Audebert A, Lehmann-Willenbrock E, Schive K, Jacobs VR (2003) Prospective clinical trial of spraygel as a barrier to adhesion formation: an interim analysis. J Am Assoc Gynecol Laparosc 10(3):339-344 